\title{
Compilação de levantamentos de uso de plantas medicinais no Rio Grande do Sul
}

\author{
| ${ }^{1}$ Roger Remy Dresch, ${ }^{2}$ Yasmin Boff Libório, ${ }^{3}$ Sílvia Beatriz Costa Czermainski |
}

Resumo: A valorizaçáo e o registro do conhecimento tradicional sobre plantas medicinais é objetivo de políticas públicas nacionais e estaduais. Nesse sentido, a Política Intersetorial de Plantas Medicinais e Fitoterápicos no Rio Grande do Sul preconiza, dentre outros aspectos, estimular a pesquisa sobre plantas medicinais, priorizando as espécies nativas. Verifica-se a reduçáo da ocorrência das espécies medicinais levando à perda do conhecimento tradicional devido à redução das áreas naturais e à desvalorização dos saberes tradicionais pelas novas geraçóes. Nesse contexto, o presente trabalho visou realizar pesquisas bibliográfica e documental sobre o uso de plantas medicinais no Rio Grande do Sul e consolidar esses estudos compondo material para a compilação de uma listagem única das espécies mais utilizadas com fins medicinais no estado. Utilizou-se metodologia quanti-qualitativa com pesquisa bibliográfica e análise documental. A compilação de dados resultou em uma lista de mais de 280 espécies vegetais distribuídas em mais de 80 famílias botânicas. Verificou-se que das 20 espécies vegetais mais usadas, menos de 50\% são nativas, fato muito relacionado à diversidade cultural do estado. Esse trabalho culminou na publicação da Relação Estadual de Plantas Medicinais de interesse do Sistema Único de Saúde no Rio Grande do Sul (REPLAME/RS).

> Palavras-chave: plantas medicinais; medicina popular; etnobotânica(o); uso medicinal; uso popular.

\author{
1 Secretaria de Estado da Saúde do \\ Rio Grande do Sul. Porto Alegre- \\ RS, Brasil (rogdresch@gmail.com). \\ ORCID: 0000-0001-9348-7011 \\ 2 Pontifíca Universidade Católica do \\ Rio Grande do Sul. Porto Alegre-RS, \\ Brasil (yasmimboff@gmail.com). \\ ORCID: 0000-0001-9291-2047 \\ ${ }^{3}$ Secretaria de Estado da Saúde do \\ Rio Grande do Sul. Porto Alegre- \\ RS, Brasil (silviaczer@gmail.com). \\ ORCID: 0000-0003-1254-7284
}

Recebido em: 15/07/2019 Aprovado em: 13/01/2020 Revisado em: 24/05/2021 


\section{Introdução}

O A valorização do conhecimento tradicional e popular sobre plantas medicinais é objetivo e diretriz de políticas públicas nacionais (BRASIL, 2006a) e estaduais (RIO GRANDE DO SUL, 2006), da preservação cultural à pesquisa, com prioridade para as espécies nativas. A sociodiversidade e a rica biodiversidade medicinal do país vêm ao encontro das reivindicaçôes da população pela Fitoterapia como opçáo terapêutica no Sistema Único de Saúde e remetem à necessidade de conhecer e registrar o uso de plantas medicinais como base para sua preservação e aproveitamento sustentável.

Movimentaçóes humanas, a introduçáo de elementos culturais exóticos, o extrativismo predatório, problemas ambientais, etc., resultam na redução da ocorrência das espécies medicinais em determinada região e na perda do conhecimento tradicional associado. O conhecimento que foi acumulado historicamente e transmitido através de inúmeras geraçôes acaba se traduzindo na diminuição do número de espécies utilizadas para tratamento das enfermidades. Atualmente, entre os fatores relacionados à perda do conhecimento sobre plantas medicinais no Brasil, encontram-se a redução das áreas naturais e a desvalorização dos saberes tradicionais pelas novas gerações, associados ao crescente acesso à medicina convencional (AMOROZO, 2002). Dessa forma, torna-se importante a realização de registros do conhecimento tradicional, antes que as espécies e o conhecimento a elas associado sejam perdidos.

Nesse contexto, o presente trabalho visou realizar uma pesquisa bibliográfica e documental sobre o uso de plantas medicinais no RS e consolidar esses estudos, compondo material para a compilação de uma listagem única das espécies mais utilizadas com fins medicinais no estado. Tal estudo partiu da ideia de que existiam vários levantamentos dispersos, científicos ou não, de produçóes do campo popular de registro do uso de plantas medicinais, de organizaçóes diversas (instituições de ensino e pesquisa como de organizações governamentais e não governamentais, religiosas, sindicais, etc.), mas que demandava a iniciativa de reunir e compilar esse material em documento único, de modo a subsidiar estudos, pesquisas e açóes em relação às plantas medicinais nativas do estado.

\section{Fontes e Métodos}

Utilizou-se metodologia quanti-qualitativa (CRESWELL, 2010), em estudo exploratório baseado em pesquisa bibliográfica e análise documental (DIEHL; 
TATIM, 2004; OLIVEIRA, 2007), para o levantamento do uso popular das plantas medicinais do Rio Grande do Sul, mediante consulta em: levantamentos etnobotânicos (CHAVES; ZANIN, 2012; MENTZ; LUTZEMBERGER; SCHENKEL, 1997); livros científicos (ALICE et al, 1995; LOPES; ALVAREZ FILHO, 1997; SIMÓES et al, 1989); anais e documentos de fóruns e encontros realizados no Rio Grande do Sul sobre plantas medicinais (ENCONTRO, 1994; FÓRUM PELA VIDA, 1998); dados disponibilizados por centros de pesquisa e organizaçóes governamentais (CASTRO; CHEMALE, 1991-1995; CORADIN; SIMINSKI; REIS, 2011; EMATER, 2007; EMBRAPA, 2011).

Este material levou à elaboração de uma lista de 293 plantas medicinais usadas pela população do Rio Grande do Sul. Foram descritas as espécies vegetais classificadas como medicinais e não aquelas medicinais cuja principal utilização seja como aromáticas e condimentares como, por exemplo, cravo, alho, cebola, canela-da-índia, louro, salsa, citronela, manjerona, alfazema, manjericão, orégano, tomilho, pimenta, limão, pêssego, figo, laranja, lima, cúrcuma, coentro, estragão, dentre outras.

A etapa seguinte consistiu no levantamento do uso popular de plantas medicinais em distintos municípios do Rio Grande do Sul, a partir da lista de 293 espécies vegetais supracitada, mediante pesquisa bibliográfica em: artigos em bases de dados (Scielo e Lilacs); livros científicos e não científicos; bibliotecas e sites de universidades (monografias de conclusão de curso; dissertaçôes e teses; publicaçôes em eventos científicos); publicaçóes sobre plantas medicinais usadas por organizaçóes populares, sindicais, religiosas, assistenciais, ONGs, grupos tradicionais (povos indígenas, quilombolas), etc. As seguintes palavras-chave foram utilizadas para a pesquisa em databases, bibliotecas e sites de universidades: plantas medicinais, medicina popular, etnobotânica(o), uso medicinal, uso popular.

O nome científico válido das plantas medicinais foi pesquisado e confirmado em bases de dados como o site da Lista de Espécies da Flora do Brasil (http:// floradobrasil.jbrj.gov.br) com complementaçóes pelo site Tropicos (http://www. tropicos.org) e pelo site da Flora Digital do Rio Grande do Sul e de Santa Catarina (http://www.ufrgs.br/fitoecologia/florars/), quando necessário. Essa padronização foi feita em virtude de que o site da Flora Digital do Rio Grande do Sul e de Santa Catarina ainda não está concluído, além das discrepâncias entre nomes científicos de mesmas espécies vegetais em relação ao site da Lista de Espécies da Flora do Brasil. 


\section{Resultados e Discussão}

Nas últimas décadas alguns fatores têm contribuído para a utilização crescente de plantas medicinais pela população brasileira, mesmo em camadas sociais que até entâo não as usavam: o alto custo dos medicamentos industrializados, as oscilaçôes da economia, o difícil acesso da população à assistência médica e farmacêutica, bem como uma tendência generalizada dos consumidores em utilizar, preferencialmente, produtos de origem natural (SIMÓES et al, 1989).

A RDC 26, de 13 de maio de 2014, trouxe uma inovação no conceito de fitoterápicos, com a introdução do conceito de produto tradicional fitoterápico e a denominação de medicamento fitoterápico ao invés de apenas fitoterápico. Conforme essa resolução, são considerados medicamentos fitoterápicos aqueles obtidos com emprego exclusivo de matérias-primas ativas vegetais cuja segurança e eficácia sejam baseadas em evidências clínicas e que sejam caracterizados pela constância de sua qualidade. Por outro lado, são considerados produtos tradicionais fitoterápicos os obtidos com emprego exclusivo de matérias-primas ativas vegetais cuja segurança e efetividade sejam baseadas em dados de uso seguro e efetivo por um período mínimo de 30 anos ou mediante publicação na literatura técnico-científica e que sejam concebidos para serem utilizados sem a vigilância de um médico para fins de diagnóstico, de prescrição ou de monitorização (BRASIL, 2014).

No Brasil, cerca de $82 \%$ da populaçáo brasileira emprega produtos à base de plantas medicinais nos seus cuidados com a saúde, seja pelo conhecimento tradicional na medicina indígena, quilombola, entre outros povos e comunidades tradicionais, seja pelo uso na medicina popular, de transmissão oral entre geraçôes, ou nos sistemas oficiais de saúde, como prática de cunho científico, orientada pelos princípios e diretrizes do Sistema Único de Saúde (SUS) (BRASIL, 2012). Embora o Brasil seja um país tradicional no uso de plantas medicinais, ainda não existem estudos específicos para essa vertente de mercado que avaliem estatisticamente seu crescimento e o consumo da população (CASTRO; ALBIERO, 2016). Contudo, ao nível global, segundo a Organização Mundial da Saúde, cerca de $80 \%$ da população mundial depende da medicina tradicional para as suas necessidades primárias de cuidado à saúde. Nesse sentido, a maior parte envolve a utilização de extratos de plantas ou os seus princípios ativos. Desse total, pelo menos 30\% deram-se por indicação médica (VENTURA, 2012; WHO/IUCN/WWF, 1986). 
Por outro lado, de acordo com a Associação Brasileira das Empresas do Setor Fitoterápico, Suplemento Alimentar e de Promoção da Saúde (Abifisa), o mercado de fitoterápicos brasileiro em 2015 apresentou crescimento em torno de 8\% comparado ao mesmo período de 2014, em que já havia crescido 6,1\% em relação ao ano anterior, ou seja, o segmento vem ampliando o patamar de crescimento (FEBRAFAR, 2016).

Segundo dados do IMS Health, os produtos fitoterápicos representam $2 \%$ do faturamento do mercado total de medicamentos, movimentando R \$ 1,6 bilhão em 2015 no Brasil (FEBRAFAR, 2016). Em que pese o crescimento do setor registrado nos últimos anos, estima-se que apenas $10 \%$ dos brasileiros consumam regularmente medicamentos fitoterápicos. Em parte, isso se explica pelo fato de o mercado local ser ainda recente em comparação ao europeu e asiático, que consomem o produto há muitos anos. Outro motivo está relacionado à desinformação sobre essa categoria de medicamentos pela população e pelos profissionais prescritores (TERRA JUNIOR; MALDONADO; ARNOBIO, 2015).

A adoção de nomes de medicamentos ou fármacos para denominar plantas medicinais pela população, somada às dificuldades de identificação, abre possibilidades para uso de espécies vegetais distintas daquela de interesse pertencentes ao mesmo gênero botânico ou não, e que não tenham a eficácia pretendida. É importante ressaltar que a literatura classifica como matéria estranha não somente a utilização de outras espécies vegetais, mas também diferentes partes botânicas da mesma espécie e que não apresentam os constituintes químicos responsáveis pelo efeito medicinal ou então com teores bem abaixo do necessário para exercerem a atividade terapêutica pretendida (SIMÓES et al., 2017).

Nesse contexto, alguns exemplos significativos se destacam como as espécies vegetais Alternanthera brasiliana (L.) Kuntzee e Alternanthera dentata (Moench) Stuchlík ex R.E.Fr., conhecidas na medicina popular do RS como terramicina, penicilina, anador, melhoral. Segundo a base de dados EPPO GLOBAL (2019) e os autores RAMOS et al. (2008), a espécie $A$. brasiliana também é denominada de sempre-viva, ervanço, caaponga, perpétua-do-brasil, perpétua-do-mato. Contudo, a maioria da população não emprega estes últimos nomes populares, podendo acarretar uso de outras espécies vegetais.

Outro exemplo diz respeito à babosa, pertencente ao gênero Aloe, pois há inúmeras espécies vegetais utilizadas pela população, sendo a mais usada popularmente a espécie Aloe arborescens Mill. Entretanto, existem publicaçóes de livros preconizando 
a utilização oral da babosa para tratamento de inúmeras doenças com ausência de toxicidade por organizaçóes que utilizam as plantas na forma do conhecimento tradicional, como a Pastoral da Saúde (ZAGO, 2010; 2011). No caso da babosa, vale ressaltar o informe técnico divulgado pela ANVISA no ano de 2011 recomendando apenas o uso externo da babosa, especialmente no tratamento da pele para fins de cicatrização, fato já comprovado cientificamente (ANVISA, 2011). Estudos científicos citados pela ANVISA demonstraram risco de carcinoma colorretal pelo emprego de laxantes à base de babosa por longo prazo, além de toxicidade hepática induzida pelo consumo oral de babosa, assim como hipotireoidismo, insuficiência renal e, em doses orais muito altas, pode ocasionar inclusive ação nefrotóxica. Em que pesem esses estudos, a larga utilização pela população de formulaçôes que a contém sugere que as pesquisas de forma de uso e dessas formulaçóes devem ser aprofundadas para validação científica, além da validação tradicional.

Outro exemplo é a utilização de plantas medicinais denominadas de arnica e boldo. A Arnica montana L. é recomendada para uso externo, como antiinflamatório em contusões e distensões, além de não ser indicado o seu uso em lesôes abertas devido ao risco de provocar danos na pele (BEVILAQUA; SCHIEDECK; SCHWENGBER, 2007; BRASIL, 2021). Contudo, sabe-se que a população nem sempre tem acesso à informação segura quanto ao uso medicinal das espécies vegetais. Ainda, há inúmeras espécies de gêneros diferentes chamadas popularmente de arnica, o que torna a questáo mais complexa, podendo ocasionar utilizaçáo de uma planta medicinal equivocada por parte da população. $\mathrm{O}$ mesmo ocorre para o boldo. A espécie medicinal de referência é o boldo-do-chile (Peumus boldus Molina), planta medicinal exótica no Brasil, mas infelizmente outras espécies de boldo são utilizadas pela população como equivalentes ao boldo-do-chile, e que não apresentam os mesmos efeitos terapêuticos e podem trazer sérios riscos à saúde. Nesse sentido, o Plectranthus barbatus Andrews, chamado de falso-boldo, boldo-brasileiro ou boldo-nacional, mesmo sendo usado na medicina popular para tratamento de distúrbios gastrintestinais, tem estudos pré-clínicos demonstrando efeitos tóxicos sobre o fígado e rins de animais tratados durante apenas sete dias, além de um efeito hipotensor (COSTA, 2006). Assim, indivíduos que possuem pressão baixa e utilizam as folhas de $P$. barbatus podem ter agravamento de sua saúde. Além disso, o boldo-do-chile tem como principal uso terapêutico casos de distúrbios hepáticos, o que não coincide com o $P$. barbatus, desencadeador de hepatotoxicidade. 
Cabe ressaltar, ainda, que o fato de existirem plantas medicinais pertencentes ao mesmo gênero, mas que possuem inúmeras espécies denominadas com o mesmo nome popular, tornando a diferenciação entre as espécies ainda mais difícil pela população, pela semelhança botânica, como é o caso da carqueja (Baccharis spp.) e do guaco (Mikania spp.), aumentando o risco de uso de uma espécie vegetal diversa da desejada por parte do usuário.

Esses exemplos demonstram que há necessidade de se aproximar os conhecimentos tradicionais e científicos, para uma melhor utilização das plantas medicinais pelo sistema de saúde, como dever do Estado para com a saúde. Entretanto, o reconhecimento do valor do uso tradicional, estabelecido nas políticas sobre plantas medicinais e fitoterápicos, deve também ser objeto de pesquisa para sua validação de eficácia e ausência de toxicidade, na formulação adequada às práticas tradicionais.

Há de se considerar ainda que a Fitoterapia tradicional, que no Brasil tem forte influência indígena e africana, e que se utiliza de formulaçóes com mais de uma planta para muitas situaçôes, têm sua utilização baseada no princípio da integralidade do usuário. Ou seja, não objetiva apenas a dimensão física, mas considera a vitalidade de forma ampla, na totalidade física, mental, emocional e espiritual, de forma diversa de outros sistemas terapêuticos, seguindo também o conceito de saúde adotado pela OMS (WHO, 1998).

No presente trabalho foi realizado o levantamento do uso popular das plantas medicinais do Rio Grande do Sul, a fim de compor uma listagem consolidada de vários outros levantamentos, para subsidiar a pesquisa e sua utilização em programas de Fitoterapia. O estudo acarretou na elaboração de uma lista de 293 espécies vegetais utilizadas pela população do RS, classificadas em espécies nativas, cultivadas, naturalizadas e exóticas, distribuídas entre 86 famílias botânicas.

Em etapa subsequente, após a reunião de vasto material bibliográfico, num total de 134 citaçôes bibliográficas, foi pesquisada a citação de uso dessas 293 espécies em distintos municípios do Rio Grande do Sul, com o objetivo de buscar as espécies vegetais realmente mais utilizadas pela população para fins terapêuticos a partir de coleta e compilação de listas de uso de plantas medicinais.

Verificou-se que, das 293 espécies vegetais, 43 não foram citadas em nenhuma das 134 referências. Foi constatado ainda que das 20 plantas medicinais mais usadas, a partir da literatura consultada, 6 espécies são nativas. Por outro lado, ao 
serem avaliadas as 40 e 50 plantas medicinais mais usadas, 18 e 24 espécies são nativas, respectivamente.

A partir desses resultados pode-se verificar o uso predominante de espécies exóticas dentre as plantas medicinais mais utilizadas pela população, fato muito relacionado à diversidade cultural de nosso estado, principalmente devido à presença de negros e imigrantes europeus, com culturas próprias quanto ao cultivo de plantas medicinais. Esses resultados corroboram a meta-análise realizada por MEDEIROS, LADIO e ALBUQUERQUE (2013) que concluíram que o Pampa é o único bioma brasileiro com predomínio de uso de espécies exóticas, diferindo significativamente de todos os demais biomas do Brasil.

Constatou-se que a marcela (Achyrocline satureioides (Lam.) DC.), considerada a planta medicinal símbolo do RS (RIO GRANDE DO SUL, 2002), foi a espécie vegetal mais citada (87 citaçóes em 134 consultas). As 20 espécies nativas mais frequentemente mencionadas pela população do RS, em ordem de citação, estão descritas na tabela 1 . Além disso, dentre as 50 plantas medicinais nativas mais utilizadas pela população do RS, as famílias botânicas predominantes são Asteraceae (12) seguida de Myrtaceae (4), Fabaceae (3) e Verbenaceae (3).

Tabela 1. Plantas medicinais nativas mais usadas no RS

\begin{tabular}{l|l|l}
\hline Família & Espécie & Nome popular \\
\hline Asteraceae & Achyrocline satureioides (Lam.) DC. & Marcela \\
Celastraceae & Monteverdia ilicifolia (Mart. ex Reissek) Biral* & Espinheira-santa \\
Asteraceae & Baccharis crispa Spreng.* & Carqueja \\
Fabaceae & Bauhinia forficata Link & Pata-de-vaca \\
Myrtaceae & Eugenia uniflora L. & Pitangueira \\
Salicaceae & Casearia sylvestris Sw. & Erva-de-bugre \\
Malvaceae & Luehea divaricata Mart. \& Zucc. & Açoita-cavalo \\
Phyllanthaceae & Phyllanthus niruri L. & Quebra-pedra \\
Lamiaceae & Cunila microcephala Benth. & Poejo \\
Brassicaceae & Coronopus didymus (L.) Sm. & Mastruço \\
Lamiaceae & Ocimum carnosum (Spreng.) Link \& Otto ex Benth. & Alfavaca
\end{tabular}

continua... 


\begin{tabular}{l|l|l}
\hline Família & Espécie & Nome popular \\
\hline Plantaginaceae & Plantago australis Lam. & Tansagem \\
Malvaceae & Sida rhombifolia L. & Guanxuma \\
Verbenaceae & Stachytarpheta cayennensis (Rich.) Vahl & Gervão \\
Verbenaceae & Aloysia gratissima (Gillies \& Hook.) Tronc. & Erva-santa \\
Myrtaceae & Campomanesia xanthocarpa (Mart.) O.Berg & Guabiroba \\
Verbenaceae & Lippia alba (Mill.) N.E.Br. ex P. Wilson & Sálvia-da-gripe \\
Asteraceae & Mikania laevigata Sch.Bip. ex Baker & Guaco \\
Asteraceae & Baccharis articulata (Lam.) Pers. & Carqueja-miúda \\
Polygonaceae & Polygonum punctatum Elliott & Erva-de-bicho \\
\hline
\end{tabular}

Fonte: Elaborada pelos autores.

*Baccharis trimera e Maytenus ilicifolia (duas das plantas medicinais mais citadas pela populaçáo do RS), conforme descrito em REFLORA (2020) e em TROPICOS (2020), são sinônimos de Baccharis crispa e de Monteverdia ilicifolia, respectivamente, e de acordo com REFLORA (2020), estes últimos passam a ser os nomes científicos aceitos.

Em 2009, o Ministério da Saúde elaborou a RENISUS (Relação de Plantas Medicinais de Interesse ao SUS), uma lista com 71 espécies com potencial terapêutico, com o objetivo de orientar a cadeia produtiva e estimular o desenvolvimento de pesquisas científicas com plantas medicinais no Brasil. Por outro lado, ao se analisar detalhadamente a RENISUS, verifica-se que ela é composta realmente de 88 espécies vegetais e de 71 gêneros. Portanto, essa lista apresenta uma quantidade ainda mais ampla de plantas medicinais que possuem potencial para gerar produtos de interesse ao SUS. Comparando-se o presente trabalho com a RENISUS e com a RENAME (Relação Nacional de Medicamentos Essenciais), verificou-se que das 71 espécies vegetais mais usadas no RS, 30 estão presentes na RENISUS, 4 constam da RENAME e 37 são nativas. Contudo, da lista de 50 espécies nativas mais utilizadas no estado do Rio Grande do Sul, apenas 14 estão na RENISUS e 2 na RENAME.

A Política Nacional de Práticas Integrativas e Complementares (PNPIC) no Sistema Único de Saúde (BRASIL, 2006b) busca incentivar uma melhoria nos serviços públicos de saúde, inserindo diferentes abordagens e com opçóes preventivas e terapêuticas, dentre elas a Fitoterapia, onde estão incluídas as Farmácias Vivas, instituídas pela RDC 18, de 3 de abril de 2013. Consideram-se 
farmácias vivas aquelas que realizam as etapas de cultivo, coleta, processamento, armazenamento de plantas medicinais, preparação e dispensação de produtos magistrais e oficinais de plantas medicinais e fitoterápicos oriundos de horta ou horto oficial ou comunitário a serem dispensados no âmbito do SUS, não sendo permitida sua comercialização (BRASIL, 2013).

Através das Farmácias Vivas pode-se levar às comunidades dois níveis de atendimento na área de fitoterapia: produção de fitoterápicos, prescrição e dispensação na rede pública de saúde, SUS, e orientação sobre o uso correto de plantas medicinais e preparaçôes caseiras, com garantia de eficácia, segurança e qualidade, através de apoio social farmacêutico, baseado em hortos medicinais constituídos de plantas medicinais com certificação botânica (PEIXE, 2010). Cabe ressaltar que o objetivo das Farmácias Vivas é de preservar o conhecimento tradicional e as espécies nativas da regiáo, além de suprir a carência de alternativas para a melhoria da saúde da população e integração dos conhecimentos populares aos resultados de pesquisas acadêmicas com adequação às diferenças regionais.

O modelo de Farmácias Vivas traz a possibilidade de o poder público promover a disponibilização de drogas vegetais e a manipulação de medicamentos fitoterápicos em farmácias municipais, atendendo as necessidades da população, além de reduzir de forma significativa os custos, ao invés de adquirir medicamentos industrializados através de licitação. Essa iniciativa pública inclusive torna mais ágil a dispensação de medicamentos à população, em contrapartida ao desabastecimento frequente de medicamentos na atenção básica em saúde. As Farmácias Vivas também seguem as diretrizes da Política Nacional de Medicamentos (BRASIL, 1998) e Política Nacional de Assistência Farmacêutica (BRASIL, 2004) que preconizam, dentre outros aspectos, a promoção do uso racional de medicamentos e o acesso da população a serviços e produtos seguros, eficazes e com qualidade.

\section{Consideraçóes finais}

Este trabalho culminou na publicação da Relação Estadual de Plantas Medicinais de interesse do Sistema Único de Saúde no Rio Grande do Sul (REPLAME/RS), cujos objetivos são: subsidiar a formulação das relaçôes de plantas medicinais de programas de Fitoterapia municipais no estado, como parte dos instrumentos de planejamento da assistência farmacêutica municipal; subsidiar a seleção de plantas medicinais e de 
fitoterápicos em Farmácias Vivas para sua inserção nos programas de Fitoterapia; servir de base para a Agenda Estadual de Prioridades de Pesquisa em Saúde, no que se refere a plantas medicinais tanto para sua utilização como chá medicinal, como também para projetos de inovação farmacêutica (RIO GRANDE DO SUL, 2017).

\section{Referências}

ALICE, C. B. et al. Plantas Medicinais de uso popular: atlas farmacognóstico. Canoas: Ulbra, 1995.

AMOROZO, M. C. M. Uso e diversidade de plantas medicinais em Santo Antonio do Leverger, MT, Brasil. Acta Botanica Brasilica, v.16, n.2, p.189-203, 2002.

ANVISA, Informe Técnico n. 47, de 16 de novembro de 2011. 7p.

BEVILAQUA, G. A. P.; SCHIEDECK, G.; SCHWENGBER, J. E. Identificação e tecnologia de plantas medicinais da flora de clima temperado. Pelotas: Embrapa, 2007.

BRASIL. Agência Nacional de Vigilância Sanitária. Formulário de Fitoterápicos da Farmacopeia Brasileira. 2. ed. Brasília: Anvisa, 2021.

. Ministério da Saúde. Agência Nacional de Vigilância Sanitária. Resolução de Diretoria Colegiada - RDC n. 18, de 03 de abril de 2013. Dispóe sobre as boas práticas de processamento e armazenamento de plantas medicinais, preparação e dispensação de produtos magistrais e oficinais de plantas medicinais e fitoterápicos em farmácias vivas no âmbito do Sistema Único de Saúde (SUS). Diário Oficial da União, Brasília, DF, 5 abr. 2013. Seção I, p. 67.

. Ministério da Saúde. Agência Nacional de Vigilância Sanitária. Resolução de Diretoria Colegiada - RDC n. 26, de 13 de maio de 2014. Dispóe sobre o registro de medicamentos fitoterápicos e o registro e a notificação de produtos tradicionais fitoterápicos. Diário Oficial da União, Brasília, DF, 14 mai. 2014. Seção I, p. 52.

. Ministério da Saúde. Conselho Nacional de Saúde. Resolução n. 338, de 06 de maio de 2004. Aprova a Política Nacional de Assistência Farmacêutica. Diário Oficial da Uniáo, Brasília, DF, 20 mai. 2004. Seção I, p. 52.

. Ministério da Saúde. Portaria n. 3.916, de 30 de outubro de 1998. Aprova a Política Nacional de Medicamentos. Diário Oficial da União, Brasília, DF, 10 nov. 1998. Seção I, p. 18.

Ministério da Saúde. Portaria n. 971, de 03 de maio de 2006. Aprova a Política Nacional de Práticas Integrativas e Complementares (PNPIC) no Sistema Único de Saúde. Diário Oficial da União, Brasília, DF, 04 mai. 2006b. Seção I, p. 20.

- Ministério da Saúde. Secretaria de Atenção à Saúde. Departamento de Atenção Básica. Práticas integrativas e complementares: plantas medicinais e fitoterapia na Atenção Básical Ministério da Saúde. Brasília: Ministério da Saúde, 2012. 
. Presidência da República. Decreto n. 5.813, de 22 de junho de 2006. Aprova a Política Nacional de Plantas Medicinais e Fitoterápicos e dá outras providências. Diário Oficial da União, Brasília, DF, 23 jun. 2006a. Seção I, p. 2.

CASTRO, L. O.; CHEMALE, V. M. Manual de identificação e cultivo de plantas medicinais, condimentares e aromáticas. Porto Alegre: Secretaria da Educação do Estado do Rio Grande do Sul, 1991-1995.

CASTRO, R. A.; AlBIERO, A. L. M. O mercado de matérias primas para indústria de fitoterápicos. Revista Fitos, Rio de Janeiro, v.10, n.1, p.59-72, jan./mar. 2016.

CHAVES, A. S.; ZANIN, E. M. Etnobotânica em comunidades rurais de origem italiana e polonesa do município de Erechim/RS. Perspectiva, v.36, n.133, p.95-113, mar. 2012.

CORADIN, L.; SIMINSKI, A.; REIS, A. Espécies Nativas da Flora Brasileira de Valor Econômico Atual ou Potencial: Plantas para o Futuro - Região Sul. Brasília: Ministério do Meio Ambiente, 2011.

COSTA, M. C. C. D. Uso popular e açóes farmacológicas de Plectranthus barbatus Andr. (Lamiaceae): revisão dos trabalhos publicados de 1970 a 2003. Revista Brasileira de Plantas Medicinais, v.8, n.2, p.81-88, 2006.

CRESWELL, J. W. Projeto de pesquisa: métodos qualitativo, quantitativo e misto. 3. ed. Porto Alegre: Artmed, 2010.

DIEHL, A. A.; TATIM, D. C. Pesquisa em ciências sociais aplicadas. São Paulo: Prentice Hall, 2004.

EMATER. Plantas medicinais, aromáticas e condimentares. Porto Alegre, RS: EMATER/RSASCAR, 2007.

EMBRAPA. Plantas Medicinais indicadas pela Anvisa. Pelotas: Embrapa Clima Temperado, 2011. Folheto. 8p.

ENCONTRO ESTADUAL SOBRE PLANTAS MEDICINAIS, 1., 1994, Porto Alegre. Revista Fepagro, n.1, jan. 1996.

EPPO GLOBAL DATABASE. Abr. 2019. Disponível em: <https://gd.eppo.int/>. Acesso em: 25 abr. 2019.

FEBRAFAR. Febrafar fala sobre fitomedicamentos no Guia das Farmácias. Febrafar. Out. 2016. Disponível em: <https://www.febrafar.com.br/febrafar-fitomedicamentosfarmacias/\#:-:text=Desempenho\%20dos\%20fitos,em\%20rela\%C3\%A7\%C3\%A3o\%20 ao\%20ano\%20anterior>. Acesso em: 20 mai. 2021.

FÓRUM PELA VIDA - PROJETO PLANTAS VIVAS. Porto Alegre: Assembleia Legislativa, 1998. 
LOPES, A. M. V.; ALVAREZ FILHO, A. Plantas usadas na medicina popular do Rio Grande do Sul. Santa Maria: Infograph, 1997.

MEDEIROS, P. M.; LADIO, A.; ALBUQUERQUE, U. P. Patterns of medicinal plant use by inhabitants of brazilian urban and rural areas: a macroscale investigation based on available literature. Journal of Ethnopharmacology, v. 150, p. 729-746, 2013.

MENTZ, L. A.; LUTZEMBERGER, L. C.; SCHENKEL, E. P. Da Flora Medicinal do Rio Grande do Sul. Caderno de Farmácia, v.13, n.1, p.25-48, 1997.

OLIVEIRA, M. M. de. Como fazer pesquisa qualitativa. Petrópolis: Vozes, 2007.

PEIXE, J. C. O. Estudo da viabilidade econômica do programa farmácia viva do município de Maracanaú, Ceará. 2010. 70 p. Trabalho de Conclusão de Curso (Especialização em Gestão da Assistência Farmacêutica), Escola de Saúde Pública do Ceará, Fortaleza, 2010.

RAMOS, A. J. K. et al. Plantas com potencialmedicinal na Floresta Nacional de Canela e comunidades do entorno, Canela, Rio Grande do Sul. Porto Alegre: EMATER/RS-ASCAR, 2008.

REFLORA. Lista de Espécies da Flora do Brasil. Nov. 2020. Disponível em: http://floradobrasil.jbrj.gov.br>. Acesso em: 25 nov. 2020.

RIO GRANDE DO SUL. Lei n. 11.858, de 5 de dezembro de 2002. Institui a planta medicinal símbolo do estado do Rio Grande do Sul e dá outras providências. Diário Oficial do Estado do Rio Grande do Sul, Porto Alegre, RS, 06 dez. 2002. p. 1.

. Lei n. 12.560 , de 12 de julho de 2006. Institui a política intersetorial de plantas medicinais e de medicamentos fitoterápicos no estado do Rio Grande do Sul e dá outras providências. Diário Oficial do Estado do Rio Grande do Sul, Porto Alegre, RS, 13 jul. 2006. p. 3. . Portaria n. 588, de 22 de dezembro de 2017. Institui a Relação Estadual de Plantas Medicinais de interesse do Sistema Único de Saúde no Rio Grande do Sul e listas complementares. Diário Oficial do Estado do Rio Grande do Sul, Porto Alegre, RS, 22 dez. 2017. p. 168.

SIMÓES, C. M. O. et al. Plantas da Medicina Popular no Rio Grande do Sul. 3.ed. Porto Alegre: Editora da Universidade/UFRGS, 1989.

, C. M. O. et al. Farmacognosia: do produto natural ao medicamento. Porto Alegre: Artmed, 2017.

TERRA JUNIOR, O. N.; MALDONADO, J. V.; ARNOBIO, A. Estudo do desempenho comercial dos insumos farmacêuticos vegetais sob a ótica do Comércio Exterior. Revista Fitos, Rio de Janeiro, v.9, n.3, p.233-246, jul./set. 2015.

TROPICOS. Missouri Botanical Garden. Nov. 2020. Disponível em: <http://www.tropicos 


\section{Abstract}

\section{Compilation of surveys of medicinal plants used in Rio Grande do Sul}

The appreciation and recording of traditional knowledge about medicinal plants is the aim of state and national public policies. The Intersectoral Policy of Medicinal Plants and Herbal Medicines in Rio Grande do Sul recommends the stimulation of research on medicinal plants, giving priority to native species. There is a reduction in the occurrence of medicinal species leading to the loss of traditional knowledge due to the reduction of natural areas and the devaluation of traditional knowledge by the new generations. This study aimed to perform bibliographical and documentary research on the use of medicinal plants in Rio Grande do Sul and to consolidate these studies in order to compose material for the compilation of a single list of the most commonly used plant species for medicinal purposes in the state. It was used a quantiqualitative methodology with bibliographical research and document analysis. Data collection resulted in a list of more than 280 plant species distributed in more than 80 botanical families. It was found that of the 20 species most commonly used, less than $50 \%$ are native, a fact closely related to the cultural diversity of the state. This work culminated in the publication of the State Relation of Medicinal Plants of interest to the Brazilian Unified Health System in the Rio Grande do Sul (REPLAME/RS).

Keywords: medicinal plants; popular medicine; ethnobotanic; medicinal use; popular use. 